\title{
EDITORIAL
}

\section{The potential role of 'non-rheumatic' therapies in rheumatic disease}

\author{
Elena M Massarotti ${ }^{1}$ and Daniel H Solomon ${ }^{1,2^{*}}$ \\ See related research by Ormseth et al., http://arthritis-research.com/content/15/5/R110
}

\begin{abstract}
The relationship between inflammation and insulin resistance is complex and not fully understood. Patients with rheumatoid arthritis are at increased risk of mortality from cardiovascular disease, which is known to be associated with insulin resistance. In the previous issue of Arthritis Research \& Therapy, Ormseth and colleagues report the results of an 8-week trial of pioglitazone, an agent commonly used to treat type 2 diabetes mellitus, upon the DAS-28 (disease activity score using 28 joint counts). Modest improvements in the DAS-28 CRP (DAS-28 C-reactive protein) were shown, with no effect on DAS-28 ESR (DAS-28 erythrocyte sedimentation rate). Other variables that improved with pioglitazone were the CRP, IL-6, and patient-reported assessment of global health. The authors discuss the contribution of insulin resistance to the inflammation noted in rheumatoid arthritis.
\end{abstract}

Disease-modifying anti-rheumatic drugs (DMARDs) have effectively reduced long-term disability and structural disease associated with rheumatoid arthritis (RA). However, patients with RA continue to suffer from a variety of extra-articular manifestations, including cardiovascular disease (CVD). The reasons for this risk, which is associated with increased mortality, are not fully understood. In addition to traditional CVD risk factors, there is evidence to suggest that chronic inflammation induced by cytokine-mediated inflammatory pathways increases risk, perhaps by affecting endothelial function or insulin resistance or both. The relationship between insulin resistance and inflammation is not fully elucidated; inflammation is

\footnotetext{
* Correspondence: dsolomon@partners.org

'Division of Rheumatology, Immunology and Allergy, Brigham and Women's Hospital and Harvard Medical School, 75 Francis Street, Boston, MA 02115, USA 2Division of Pharmacoepidemiology, Brigham and Women's Hospital and Harvard Medical School, 75 Francis Street, Boston, MA 02115, USA
}

known to be associated with insulin resistance, and hyperinsulinemia is also associated with inflammation.

In the previous issue of Arthritis Research \& Therapy, Ormseth and colleagues [1] further explore the relationship between inflammation, insulin resistance, and RA by studying the effects of 8 weeks of pioglitazone, a thiazolidinedione used to treat type 2 diabetes mellitus, in a double-blinded, placebo-controlled, crossover study of 34 subjects with 'moderate disease activity' due to RA. The study population consisted largely of non-obese females with a mean baseline C-reactive protein (CRP) of 4.58 and DAS-28 CRP (disease activity score using 28 joint countsCRP) of more than 4.4. Over half of the subjects were taking corticosteroids at study entry; $70 \%$ were taking methotrexate, and over half were taking 'biologic' therapy (class not specified). Erosive disease was present in the majority. The study was conducted at a single center and lasted for 3 years. The primary outcome measure was the change in the DAS-28; secondary outcome measures were individual components of the DAS-28, IL-6 (pg/mL), tumor necrosis factor-alpha $(\mathrm{pg} / \mathrm{mL})$, change in the modified health assessment questionnaire, and the homeostatic model assessment (HOMA) for insulin resistance, a standard measurement of insulin resistance. Treatment with pioglitazone resulted in a $9 \%$ improvement in the DAS-28 CRP $(P=0.046)$ but had no effect on the DAS-28 ESR (DAS-28 erythrocyte sedimentation rate) $(0.6 \%, P=0.92)$. Other significant results were a nearly $50 \%$ reduction in CRP $(\mathrm{mg} / \mathrm{dL})(P<0.001)$, an approximately $70 \%$ reduction in IL-6 $(P=0.01)$, and an $11 \%$ improvement in the patient-reported visual analogue scale for global health. As expected, the HOMA improved by $26 \%$ ( $95 \%$ confidence interval $-43.8 \%$ to $-3.7 \%$ ).

Results from this small but provocative study underscore the complex interplay between inflammation and insulin resistance. The parallel improvements in IL-6 and insulin resistance are in keeping with the known, strong association between insulin resistance and IL-6 [2]. The reductions in IL-6, CRP, and DAS-28 CRP in the study by 
Ormseth and colleagues highlight the known inhibitory effects of pioglitazone on pro-inflammatory cytokines [3]. The improvements in DAS-CRP, and not DAS-ESR, suggest that the independent effects of pioglitazone on IL-6 may have accounted for much of the reduction in disease activity, further suggested by the lack of improvement in the tender and swollen joint counts. This link between IL-6 inhibition and insulin resistance was also observed in an open-label study of tocilizumab, an inhibitor of IL-6, in 11 non-diabetic subjects with steroid-dependent, stable RA; the HOMA significantly improved at 12 weeks [4].

Examining the RA treatment effects of agents approved for other indications like diabetes or hyperlipidemia, known risk factors for CVD, is not new. In the Trial of Atorvastatin Rheumatoid Arthritis, a randomized study of atorvastatin in patients with active RA, investigators demonstrated modest improvements in the DAS-28, along with reductions in IL-6 and CRP [5]. The authors suggest considering the use of atorvastatin as an adjunct to DMARD therapy in an effort to mitigate disease activity and provide 'vascular' protection. Although the use of atrovastatin and pioglitazone as primary RA treatments is not justified, use of these agents in patients with RA and hyperlipidemia or type 2 diabetes mellitus may be worthwhile. Studies such as these, along with studies of biological agents in RA that affect inflammatory cytokine pathways, provide insights into why patients with RA are at increased risk of CVD. It is likely that more complete immunosuppression will reduce CVD risk but that adjunctive therapy with other agents like statins and thiazolidinediones, which possess immunomodulatory effects, may have a role in future RA therapeutic regimens. In the future, the 'complete' treatment of RA will include elimination of the direct effects of uncontrolled inflammation on joints as well as mitigation of CVD and diabetes, which appear to be related to the ongoing inflammatory process.

\section{Abbreviations}

CRP: C-reactive protein; CVD: Cardiovascular disease; DAS-28: Disease activity score using 28 joint counts; DMARD: Disease-modifying anti-rheumatic drug; ESR: Erythrocyte sedimentation rate; HOMA: Homeostatic model assessment; IL: Interleukin; RA: Rheumatoid arthritis.

\section{Competing interests}

DHS receives research support from Amgen (Thousand Oaks, CA, USA), Eli Lilly and Company (Indianapolis, IN, USA), Pfizer (New York, NY, USA), and Corrona (Albany, NY, USA) and serves in unpaid roles on studies funded by Pfizer, Novartis (Basel, Switzerland), Bristol-Myers Squibb (New York, NY, USA), and Eli Lilly and Company. EMM has received honoraria $(<\$ 10,000)$ from Amplimmune (Gaithersburg, MD, USA) and Sanofi (Paris, France) and is an investigator for lupus trials sponsored by Human Genome Sciences

(Rockville, MD, USA) and Bristol-Myers Squibb.

\section{Acknowledgments}

DHS is supported by a mentoring award from the National Institutes of Health (K24 AR055989).

\section{References}

1. Ormseth MJ, Oeser AM, Cunningham A, Bian A, Shintani A, Solus J, Tanner SB, Stein CM: Perixisome proliferator-activated receptor gamma agonist effect on rheumatoid arthritis: a randomized controlled trial. Arthritis Res Ther 2013, 15:R110.

2. Fernandez-Real JM, Vayreda M, Richart C, Broch M, Vendrell J, Ricart W: Circulating interleukin- 6 levels, blood pressure, and insulin sensitivity in apparently healthy men and women. J Clin Endocrinol Metab 2001, 86:1154-1159.

3. Zhang WY, Schwartz EA, Permana PA, Reaven PD: Pioglitazone inhibits the expression of inflammatory cytokines from both monocytes and lymphocytes in patients with impaired glucose tolerance. Arterioscler Thromb Vase Biol 2008, 28:2312-2318.

4. Schultz O, Oberhauser F, Saech J, Rubbert-Roth A, Hahn M, Krone W, Laudes M: Effects of inhibition of interleukin- 6 signaling on insulin sensitivity and lipoprotein (a) levels in human subjects with rheumatoid diseases. PLoS One 2010, 5:el4328.

5. McCarey DW, McInnes IB, Madhok R, Hampson R, Scherbakov O, Ford I, Capell HA, Sattar N: Trial of atorvastatin rheumatoid arthritis (TARA): double-blind, randomised placebo-controlled trial. Lancet 2004 363:2015-2021.

\subsection{6/ar4371}

Cite this article as: Massarotti and Solomon: The potential role of 'nonrheumatic' therapies in rheumatic disease. Arthritis Research \& Therapy 2013, 15:124 